\title{
Design and Evaluation of Gemini Surfactant-Based Lipoplexes Modified with Cell-Binding Peptide for Targeted Gene Therapy in Melanoma Model
}

\author{
Waleed Mohammed-Saeid ${ }^{1,2}$, Rania Soudy ${ }^{3}$, Richa Tikoo ${ }^{1}$, Kamaljit Kaur ${ }^{4}$, Ronald Verrall ${ }^{5}$, Ildiko Badea ${ }^{1}$ \\ ${ }^{1}$ Drug Design and Discovery Research Group, College of Pharmacy and Nutrition University of Saskatchewan, Saskatoon, \\ Canada. ${ }^{2}$ College of Pharmacy, Taibah University, Medina, Saudi Arabia. ${ }^{3}$ Department of Medicine in the Faculty of \\ Medicine and Dentistry, University of Alberta, Edmonton, Canada. ${ }^{4}$ Chapman University School of Pharmacy (CUSP), \\ Harry and Diane Rinker Health Science Campus, Chapman University, Irvine, California, USA. ${ }^{5}$ Department of Chemistry, \\ University of Saskatchewan, Saskatoon, Canada.
}

Received, June 8, 2018; Revised, August 23, 2018; Accepted, September 16, 2018; Published, September 16, 2018.

\begin{abstract}
Purpose Achieving successful gene therapy requires delivery of a gene vector specifically to the targeted tissue with efficient expression and a good safety profile. The objective of this work was to develop, characterize and determine if a novel gemini surfactant-based lipoplex systems, modified with a cancer-targeting peptide p18-4, could serve this role. Methods The targeting peptide p18-4 was either chemically coupled to a gemini surfactant backbone or physically co-formulated with the lipoplexes. The influence of targeting ligand and formulation strategies on essential physicochemical properties of the lipoplexes was evaluated by dynamic light scattering and small angle X-ray scattering techniques. In vitro transfection activity and cellular toxicity of lipoplexes were assessed in a model human melanoma cell line. Results All lipoplexes zeta potential and particle size were optimal for cellular uptake and physical stability of the system. The lipoplexes adopted an invertedhexagonal lipid arrangement. The lipoplexes modified with the peptide showed no significant changes in physicochemical properties or lipoplex assembly. The modification of the lipoplexes with the targeting peptide significantly enhanced protein expression 2-6 fold compared to non-modified lipoplexes. In addition, p18-4 modified lipoplexes significantly improved the safety of the lipoplexes. The ability of the p18-4 modified lipoplexes to selectively express the model protein was confirmed by using healthy human epidermal keratinocytes (HEKa). Conclusion The gemini surfactant-based lipoplexes modified with p18-4 peptide showed significantly higher efficiency and safety compared to the system that did not contain a cancer targeting peptide and provided evidence for their potential application to achieve targeted melanoma gene therapy.
\end{abstract}

\section{INTRODUCTION}

In gene therapy, the efficiency of non-viral vectors depends on the successful delivery and expression of the exogenous genetic material into the targeted tissue, while causing minimum toxicity. For cancer gene therapy, non-viral gene delivery systems, which usually assemble as nano-sized particles, have the ability to non-specifically target the tumor (passive targeting) by an enhanced permeability and retention (EPR) effect, extravasation through the leaky vasculature surrounding the tumors and entrapment in the tumor mass.(1) However, passive cancer targeting can be challenged by several factors including, but not limited to, the type of cancer, the heterogeneity of the tumor, blood supply, the interaction of the nanoparticles with the tumor and surrounding tissue, and the characteristics of the delivery system. $(2,3)$. On the other hand, cancer-specific targeting can be achieved by decorating the non-viral delivery system with cancer-specific ligands (active targeting). Such ligands can be a small-molecule moiety, protein, peptide or antibody (monoclonal antibody or fragment) that can specifically bind to an overexpressed enzyme, a receptor or a cancerspecific antigen.(4) Such targeting can enhance the accumulation and

Corresponding Author: Ildiko Badea, Drug Design and Discovery Research Group, College of Pharmacy and Nutrition University of Saskatchewan, Saskatoon, Canada. E-mail: ildiko.badea@usask.ca 
interaction of the ligand-modified nanoparticles which can improve their specific cellular internalization. As a result of specific uptake by cancer cells, off-target gene expression and toxicity can be avoided or at least minimized.(5).

In cancer gene and drug delivery, different ligands have been explored for cancer specific targeting. These ligands include monoclonal antibodies such as trastuzumab to target HER2 receptor in breast cancer, peptides such as arginylglycyl-aspartic acid (RGD) to target overexpressed integrins, and small molecules such as folic acid to target the folate-binding protein.(3) Among different peptide ligands that have been explored for cancer targeting is p160, a linear dodecapeptide (VPWMEPAYQRFL) that was identified by phage display. It displayed a superior affinity for neuroblastoma cells and selective internalization in different breast cancer cell lines but not by normal umbilical vein/vascular endothelium cells (HUVEC).(6, 7) Based on the structure of p160, several linear decapeptides have been engineered with higher affinity to cancer cells (MDA-MB-435, and MCF-7) and better enzymatic stability.(8) Among these targeting peptides, p18-4 (WxEAAYQrFL) [Figure 1] demonstrated the highest affinity and selectivity toward breast cancer cells and showed resistance to proteolytic degradation.(8) Decoration of liposomaldoxorubicin with p18-4, as a cancer targeted drug delivery system, significantly improved the cellular uptake and cytotoxicity of doxorubicin selectivity in breast cancer.(9) In mice bearing MDA-MB-435 tumour, the anti-cancer activity of the p18-4 modified liposomal-doxorubicin was improved in comparison to the unmodified liposomal system.(10).

Cationic gemini surfactants are cationic lipids that have been comprehensively evaluated and characterized for gene delivery. $(11,12)$ The versatility in the chemical structure of gemini surfactants makes it easy to produce highly efficient gene delivery systems with a good safety profile.(1315) For example, chemical modification in the basic structure of gemini surfactant by introducing amino acid substituents in the spacer region, producing 127N(GK)-12 [Figure 1], has led to the production of an efficient biocompatible carrier. The applicability of topical gene delivery by cationic gemini surfactants was evaluated previously for dermal, vaginal and ocular applications.(13, 15, 16) The positive outcome provided evidence for the viability of using gemini surfactant-based lipoplexes for topical and transdermal gene delivery. Recently, we have reported the development and evaluation of an RGD-modified gemini surfactant lipoplex system that shows specificity to melanoma.(17) The introduction of the RGD moiety into the gemini surfactant structure enhanced the transfection activity by more than two fold compared to nonmodified lipoplexes.(17) The successful targeting of overexpressed integrin in a melanoma model provided evidence for the potential of using gemini surfactant-based lipoplexes for targeted cancer gene therapy.

The objective of this work was to develop and characterize a novel cationic gemini surfactant-based lipoplex decorated with a specific cancer-targeting ligand (p18-4) and to evaluate its activity in a melanoma model. As well, we investigated the influence of the ligand modification on the essential physicochemical properties of the lipoplex system that govern the transfection activity. The specificity of the developed ligand-modified lipoplexes to target melanoma cells was also examined.

\section{MATERIALS \& METHODS}

\section{Materials}

$\mathrm{N}, \quad \mathrm{N}$-diisopropylethylamine (DIPEA), 1-[bis (dimethylamino) methylene] -1H-1,2,3-triazolo[4,5b]pyridinium 3-oxidehexafluorophosphate (HATU), trifluoroacetic acid (TFA) and dimethyl sulfoxide (DMSO spectroscopy grade) were purchased from Sigma-Aldrich (Oakville, ON, Canada). Helper lipid 1 , 2 dioleyl - sn - glycerol phosphatidylethanolamine (DOPE) was purchased from Avanti Polar Lipids, (Alabaster, AL, USA). 3(4,5-Dimethylthiazol-2-yl)-2,5-diphenyltetrazolium bromide (MTT) was purchased from Invitrogen Corporation (Grand Island, NY, USA). All solvents used were HPLC grade.

Synthesis of gemini surfactant and peptide p18-4 Synthesis and characterization of $12-7 \mathrm{~N}(\mathrm{GK})-12$ gemini surfactant was reported previously.(18) Synthesis and characterization of linear peptide $\mathrm{p} 18$ 4 were described previously.(9) 
a)

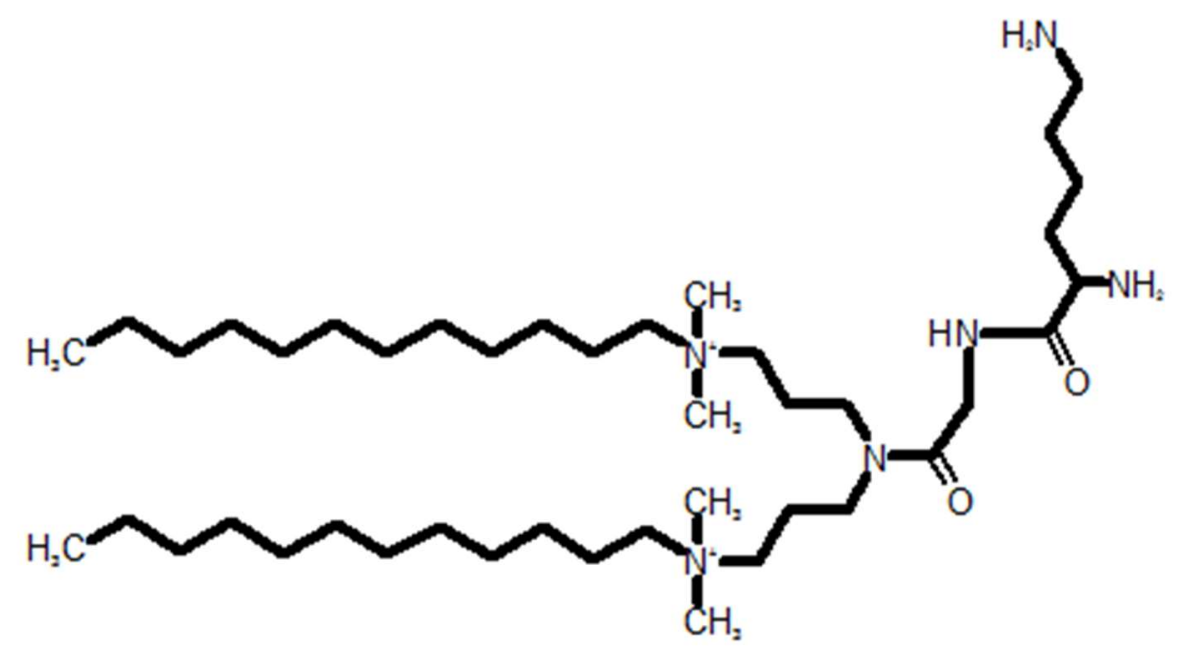

b)<smiles>CCC[C@H](NC(=O)[C@H]([NH3+])Cc1c[nH]c2ccccc12)C(=O)N[C@@H](CCC(=O)O)C(=O)N[C@@H](C)C(=O)N[C@@H](C)C(=O)N[C@@H](Cc1ccc(O)cc1)C(=O)N[C@@H](CCC(N)=O)C(=O)N[C@@H](CCCNC(=N)N)C(=O)N[C@@H](Cc1ccccc1)C(=O)NC(CC(C)C)C(=O)O</smiles>

c)<smiles>CCCCCCCCCCCCCCCCCCN(C)CCCN(CCC[N+](C)(C)CCCCCCCCC)C(=O)CCC(=O)NC(Cc1c[nH]c2ccccc12)C(=O)NC(CCC)C(=O)NC(CCC(=O)O)C(=O)NC(C)C(=O)NCC(=O)NC(Cc1ccc(O)cc1)C(=O)NC(CCC(N)=O)C(=O)NC(CCCCNC(=N)N)C(=O)NC(Cc1ccccc1)C(=O)NC(CC(C)C)C(=O)O</smiles>

Figure 1. Chemical structure of a) 12-7N(GK)-12, b) p18-4 peptide [p18-4] (WxEAAYQrFL) and c) 12-7N(p18-4)-12 gemini surfactant [p18-4-G] 


\section{Synthesis of p18-4 modified gemini surfactant}

Peptide p18-4 on the chlorotrityl resin (with all side chain groups protected with Boc) was provided by Dr. Kaur's group. (8) The synthesis of gemini surfactant backbone [3-(bis 3-(N-dodecyl-N,Ndimethylamino)propyl)carbamoyl propanoic acid $]^{2+}$ $2 \mathrm{Cl}^{-}$was described previously.(19) In brief, under a $\mathrm{N}_{2}$ atmosphere using standard Schlenk techniques, a Schlenk flask equipped with a magnetic stir bar was charged with 3,3' - iminobis ( N , N dimethylpropylamine) (1.682 $\mathrm{g}, 8.989 \mathrm{mmol})$ and succinic anhydride $(0.988 \mathrm{~g}, 9.873 \mathrm{mmol})$ in $15 \mathrm{~mL}$ of DMF to form a homogeneous solution. After 3 days of stirring at ambient temperature, the reaction mixture was concentrated under vacuum to obtain an orange-yellow oily substance as the desired compound in quantitative yield. The compound was charged in a round-bottom flask with propanoic acid, (800.0 mg, $2.785 \mathrm{mmol}$ ) and 1-iodododecane (2.063 $\mathrm{g}, 1.720 \mathrm{~mL}, 6.933 \mathrm{mmol})$ in DMF (20 mL) to form a yellow homogeneous solution. After $12 \mathrm{~h}$ of stirring at ambient temperature, the solvent was removed under vacuum and the residue washed several times with diethyl ether to remove the excess iodododecane. The sample was dried, yielding an orange oily substance that was dissolved in $10 \mathrm{~mL}$ of distilled water and 2.5 equivalents of Amberlite ${ }^{\circledR}$ IRA-400(Cl) $(2.400 \mathrm{~g}, 6.933 \mathrm{mmol})$ was added to the solution. After stirring for $1 \mathrm{~h}$, the Amberlite resin was removed by filtration and the residue washed with water. An orange, oily substance [ 3 - ( bis 3 - ( $\mathrm{N}-$ dodecyl $-\mathrm{N}, \mathrm{N}$ dimethylamino)propyl)carbamoyl propanoic acid] ${ }^{2+}$ $2 \mathrm{Cl}^{-}$was obtained in almost quantitative yield after removing excess water by freeze-drying.

The gemini backbone was activated under a $\mathrm{N}_{2}$ atmosphere in a round bottom flask, by reacting [ 3 ( bis $3-(\mathrm{N}-$ dodecyl $-\mathrm{N}, \mathrm{N}$ dimethylamino $)$ propyl) carbamoyl propanoic acid $]^{2+} 2 \mathrm{Cl}^{-}$(M.W. $696.96 \mathrm{~g} / \mathrm{mol})$ with (O-(7-azabenzotriazol-1-yl)-N $, \mathrm{N}, \mathrm{N}^{\prime}, \mathrm{N}^{\prime}$-tetramethyluronium hexafluorophosphate, HATU) $(1.2 \mathrm{eq}, \mathrm{M} . W .380 .23 \mathrm{~g} / \mathrm{mol})$ and $(\mathrm{N}, \mathrm{N}-$ diisopropylethylamine, DIPEA) (2 eq, M.W. 129.25 $\mathrm{g} / \mathrm{mol}$ ) in $10 \mathrm{~mL}$ of dry DMF at room temperature for $15 \mathrm{~min}$.

For the coupling reaction, a solid phase peptide synthesis vessel [Medium Frit, GL 25 thread, Fritted Disc. Dia./25] (Chemglass Life Sciences, Vineland, NJ, USA) was charged with [p18-4]-chlorotrityl resin and activated gemini surfactant (1:5 moles) in $10 \mathrm{~mL}$ of dry DMF. The coupling reaction was carried out for 3 days with continuous shaking and the solution removed from the vessel. The peptidegemini surfactant was cleaved from the resin and all protecting groups were removed by using 50:50 TFA/DCM, 5\% TIPS at room temperature for $1 \mathrm{~h} \mathrm{(3}$ times). The solution containing the modified gemini surfactant [p18-4-G] was collected and concentrated by rotary evaporation. The product was dissolved in 50:50 (v/v) $\mathrm{H}_{2} \mathrm{O} /$ methanol and subjected to 3 cycles of cold acetone precipitation. The structure of the compound was confirmed by using an API QSTAR XL MS/MS hybrid QqToF tandem mass spectrometer equipped with an ESI source (Applied Biosystems Inc., CA, USA). ESI m/z $634[\mathrm{M}+\mathrm{H}]^{3+}$ (expected $\mathrm{m} / \mathrm{z}$ for $[\mathrm{M}+\mathrm{H}]^{3+}$ is 634.758 ).

\section{Lipoplex formulations}

The construction of the plasmid pGThCMV.IFNGFP, used as a model for a robust plasmid, was described previously.(13) Plasmid DNA (coded as $\mathbf{P}$ in lipoplexes) was isolated and purified using QIAGEN Plasmid Giga Kit (Mississauga, ON, Canada) following the manufacturer's protocol. Aqueous solutions of $3 \mathrm{mM}$ gemini surfactant/peptide were used to prepare all lipoplex formulations evaluated in this work.

Lipoplexes, Table 1, were formulated using a plasmid to gemini surfactant (phosphate/nitrogen) charge ratio of 1:10 in the presence of (DOPE) as a helper-lipid (coded as $\mathbf{L}$ in lipoplexes) creating plasmid/gemini surfactant/lipid lipoplexes [P.G.L], as described previously.(17) In brief, The DOPE were vesicles formed by sonication in $9.25 \%$ sucrose solution $(\mathrm{pH} 9)$ at a final concentration of $1 \mathrm{mM}$ DOPE and filtered through Acrodisc ${ }^{\circledR} 0.45 \mu \mathrm{m}$ syringe filters (Pall Gelman, Ann Arbor, MI). The $[\mathrm{P} / \mathrm{G}]$ lipoplexes were prepared by mixing an aliquot of $200 \mu \mathrm{g}$ pDNA aqueous solution with an appropriate amount of $3 \mathrm{mM}$ gemini surfactant solution and incubated at room temperature for 20 min. The [P.G.L] systems were prepared by mixing $[\mathrm{P} / \mathrm{G}]$ lipoplexes with the DOPE vesicles at gemini surfactant to DOPE molar ratio of 1:10 and incubated at room temperature for $20 \mathrm{~min}$.

Peptide-modified lipoplex formulations were prepared in two different ways (as described earlier)(17). Briefly, $10 \%$ of total non-modified gemini surfactant, 12-7N(GK)-12, was substituted by either peptide-modified gemini surfactant (p18-4G) in L2 and L3, (Table 1) or targeting peptide (p184) in L4 and L5 (Table 1).

\section{Size and zeta potential measurements}


Formulations ( $800 \mu \mathrm{L}$ of each) were transferred into a special cuvette (DTS1061, Malvern Instruments, Worcestershire, UK) for size distribution and zetapotential measurements using a Zetasizer Nano ZS instrument (Malvern Instruments, Worcestershire, UK). Each sample was measured four times, and the results are expressed as an average \pm standard deviation (SD) of a triplicate with corresponding polydispersity index (PDI) value (Table 2).

\section{Small-angle X-ray Scattering (SAXS) analysis}

Small-angle X-ray Scattering (SAXS) measurements were carried out at the Stanford Synchrotron Radiation Lightsource, Menlo Park, CA, California. Lipoplexes prepared as described above were concentrated (10x) by speed vacuuming at $35^{\circ} \mathrm{C}$. A wavelength of $1.1271 \AA(11 \mathrm{KeV}$ energy) was utilized. Samples were loaded in $1.5 \mathrm{~mm}$ boron-rich glass capillaries (Charles Supper Company, USA). The scattered X-ray was detected on MAR225-HE $(225 \mathrm{~mm} \times 225 \mathrm{~mm}(3072 \times 3072$ pixels, pixel size $73.24 \mu \mathrm{m})$ at $20 \mathrm{~s}$ exposure time and at a sample to detector distance of $1.1 \mathrm{~m}$. The SAXS detector was calibrated with silver behenate. GSASII software was used to plot diffraction intensity versus $2 \theta$ (where $\theta$ is the diffraction angle) or $q$ (scattering vector) by radial integration of the $2 \mathrm{D}$ patterns.

\section{Cell culture and in vitro transfection}

Human malignant melanoma (A375) cell line $\left(\right.$ ATCC $^{\circledR}$ CRL-1619 ${ }^{\mathrm{TM}}$ ) was cultured in Dulbecco's modified Eagle's medium (DMEM) supplemented with $10 \%$ fetal bovine serum and $1 \%$ antibiotic and incubated at $37{ }^{\circ} \mathrm{C}$ under an atmosphere of $5 \%$ $\mathrm{CO}_{2} / 95 \%$ air. Normal adult human epidermal keratinocyte cells (HEKa,C-005-5C, Cascade Biologics, Invitrogen), were grown on a T-75 Cell+ tissue culture flask (Sarstedt AG \& Co.) in Medium 154 (Gibco) supplemented with Human Keratinocyte Growth Supplement (HKGS, Gibco), $10 \%$ fetal bovine serum and $1 \%$ antibiotic and incubated at $37{ }^{\circ} \mathrm{C}$ under an atmosphere of $5 \%$ $\mathrm{CO}_{2} / 95 \%$ air.

The day prior to transfection, cells were seeded in 96-well tissue culture plates (BD Mississauga, ON, Canada) at a density of $1.5 \times 10^{4}$ cells/well for A375 and HEKa cell line at a density of $2 \times 10^{4}$

Table 1. Preparation methods used for formulations evaluated. All lipoplex systems prepared at 1:10 $-/+$ charge ratio and with helper lipid DOPE. P: pDNA, G:12-7N(GK)-12, L: DOPE, p18-4-G : 12-7N( p18-4)-12, p18-4: p18-4 linear peptide.

\begin{tabular}{llll}
\hline ID & Preparation of Peptide modified lipoplex & Peptide/Peptide-gemini surfactant & System produced \\
\hline L1 & Conventional & -- & [P.G.L] \\
L2 & Chemical conjugation & $10 \% 12-7 \mathrm{~N}(\mathrm{p} 18-4)-12$ & Ch[P.G.p18-4-G.L] \\
L3 & Chemical conjugation & $10 \% 12-7 \mathrm{~N}(\mathrm{p} 18-4)-12$ & Ch[P.G.L].p18-4-G \\
L4 & Physical co-administration & $10 \% \mathrm{p} 18-4$ & Ph[P.G.p18-4.L] \\
L5 & Physical co-administration & $10 \% \mathrm{p} 18-4$ & Ph[P.G.L].p18-4 \\
L6 & Chemical conjugation & $100 \% 12-7 \mathrm{~N}(\mathrm{p} 18-4)-12$ & Ch[P.G.p18-4-G.L] \\
\hline
\end{tabular}

Table 2 Physiochemical properties (size and zeta potential) of different p18-4 modified lipoplexes formulations. Size and zeta potential measurements: values are an average of $\mathrm{n}=3 \pm$ standard deviation (SD). The polydispersity (PDI) index is indicated for the size measurements as an average of three measurements \pm STD.

\begin{tabular}{lllll}
\hline Preparation Method & System produced & $\begin{array}{l}\text { Particle } \\
(\mathbf{n m}) \pm \text { SD }\end{array}$ & PDI \pm SD & $\zeta$-potential $(\mathbf{m V}) \pm$ SD \\
\hline Conventional & L1: [P.G.L] & $157 \pm(2.5)$ & $0.251 \pm(0.003)$ & $22 \pm(1.03)$ \\
Chemical conjugation & L2: Ch[P.G.p18-4-G.L] & $144 \pm(0.5)$ & $0.296 \pm(0.019)$ & $19.5 \pm(0.68)$ \\
Chemical conjugation & L3: Ch[P.G.L].p18-4-G & $145 \pm(2.4)$ & $0.268 \pm(0.002)$ & $17.7 \pm(1.45)$ \\
Physical co-administration & L4: Ph[P.G.p18-4.L] & $163 \pm(2)$ & $0.295 \pm(0.014)$ & $17 \pm(2.32)$ \\
Physical co-administration & L5: Ph[P.G.L].p18-4 & $174 \pm(2.8)$ & $0.364 \pm(0.014)$ & $15.2 \pm(1.76)$ \\
\hline
\end{tabular}


cells/well in 96-well Cell+ tissue culture plate (Sarstedt AG \& Co.). One hour before treatment, the media was replaced with non-supplemented media. Four wells per formulation were transfected with lipoplexes containing $0.2 \mu \mathrm{g}$ pGThCMV.IFN-GFP plasmid/well. Lipofectamine Plus reagent (Invitrogen Life Technologies) was used according to the manufacturer's protocol as a positive control with $0.2 \mu \mathrm{g}$ pDNA/well. The 96-well tissue culture plates were then incubated at $37{ }^{\circ} \mathrm{C}$ under $5 \% \mathrm{CO}_{2}$ and $95 \%$ air. After $5 \mathrm{~h}$, the media was removed and replaced with fresh supplemented media. Supernatants containing the secreted IFN- $\gamma$ were collected at 24 and $48 \mathrm{~h}$ and replaced with fresh supplemented media. The collected supernatants were stored at $-80{ }^{\circ} \mathrm{C}$ until further evaluated.

\section{Transfection activity (ELISA)}

Enzyme-linked immunosorbent assay (ELISA) was performed using Immulon 2 flat bottom 96-well plates (Greiner Labortechnik, Frickenhausen, Germany) following the BD Pharmingen protocol as previously described.(20) The concentration of expressed IFN- $\gamma$ was calculated from a standard IFN$\gamma$ curve using recombinant mouse IFN- $\gamma$ standard (BD Pharmingen, BD Biosciences).

\section{Cytotoxicity assay}

MTT assay was used to evaluate the cellular toxicity of lipoplex systems in A375 and HEKa cell lines. A sterile solution of $4 \mathrm{mg} / \mathrm{mL}$ of MTT in PBS buffer was prepared. Cell lines were seeded on 96-well plates and transfected with lipoplexes (as described above). After $48 \mathrm{~h}$, the cell lines were evaluated for cytotoxicity. The supplemented media was removed from the wells and replaced with $0.5 \mathrm{mg} / \mathrm{mL}$ MTT in supplemented media and incubated at $37{ }^{\circ} \mathrm{C}$ in $5 \%$ $\mathrm{CO}_{2}$ and $95 \%$ air for $3 \mathrm{~h}$. The supernatant was removed and each well washed with PBS. The purple formazan crystal that formed was dissolved in DMSO. Plates were incubated for $10 \mathrm{~min}$ at $37^{\circ} \mathrm{C}$. Absorbance was measured at $580 \mathrm{~nm}$ using BioTek microplate reader (Bio-Tek Instruments, VT, USA). The cellular toxicity is expressed as a percentage of the non-transfected control cells $\pm \mathrm{SD}$.

\section{STATISTICAL ANALYSIS}

Statistical analyses were performed using SPSS software (Version 23.0). The results are expressed as the average of three independent experiments \pm SD. One way analysis of variance (ANOVA, Dunnett's test) was used for statistical analyses. Significant differences were considered at $p<0.05$ level.

\section{RESULTS}

The influence of p18-4-modification on the physicochemical characteristics of the lipoplexes Physicochemical properties that control the transfection activity and stability of lipoplexes are: particle size, surface charge and lipid assembly.(21, 22) All lipoplexes formed particles with an average diameter of less than $180 \mathrm{~nm}$. The average particle size of the non-modified lipoplexes (L1: [P.G.L]) was $157 \pm 2.5 \mathrm{~nm}$ (Table 2). Substitution of $10 \%$ of the parent gemini surfactant [12-7N(GK)-12] with the p18-4 conjugated gemini surfactant (p18-4-G) (L2: Ch[P.G.p18-4-G.L] and L3: Ch[P.G.L].p184-G), caused a decrease in particle size to $144 \pm 0.5$ $\mathrm{nm}$ and $145 \pm 2.4$, respectively. On the other hand, physical co-formulation of free p18-4 peptide with the lipoplex system caused an increase in the average size of the assembled nanoparticles with wide poly dispersity index values. For example, the addition of $10 \%$ free p18-4 to the formed lipoplexes [P.L.G] (L5: Ph[P.G.L].p18-4) caused an increase of $17 \mathrm{~nm}$ compared to non-modified lipoplexes (L1: [P.G.L]). Zeta potential measurements indicated that all lipoplexes carry an overall positive charge in the range of $22-15 \mathrm{mV}$. The substitution of $10 \%$ of nonmodified gemini surfactant $(12-7 \mathrm{~N}(\mathrm{GK})-12)$ with p18-4 gemini surfactant or free p18-4 peptide caused a decrease in zeta potential values. For example, L5:Ph[P.G.L].p18-4 showed the most significant reduction in zeta potential value of over $30 \%$ compared to the non-modified formulations (L1).(Table 2).

The influence of the addition of the targeting peptide on the lipid phase arrangement and transitions of the lipoplexes was examined using SAXS [Figure 2]. All lipoplexes exhibited Bragg's peaks with relative ratios of $1, \sqrt{3}$ and $\sqrt{ } 4$, characteristic of an inverted hexagonal phase arrangement $\left(\mathrm{H}_{\mathrm{II}}\right)$. The preparation method and presence or absence of targeting peptide-modified gemini surfactant or free targeting peptide in the lipoplex system did not cause any alteration in the lipid phase arrangement. 


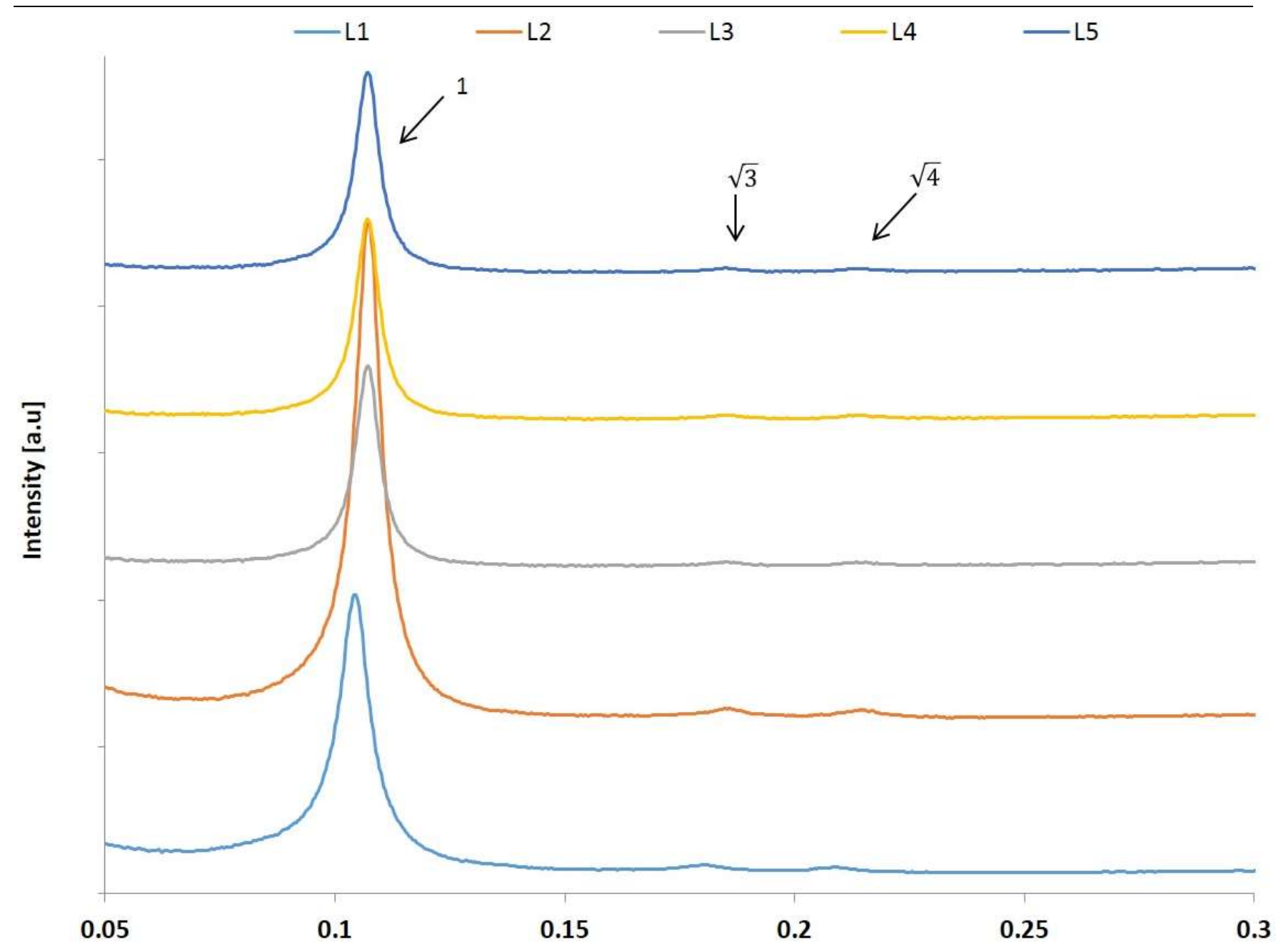

Figure 2. SAXS profile of [P.G.L] lipoplexes constructed at 1:10 -/+ charge ratio and with helper lipid DOPE (L) in all formulation. P: pDNA, G:12-7N(GK)-12, L: DOPE, p18-4-G : 12-7N( p18-4)-12, p18-4: p18-4 linear peptide.

\section{In vitro evaluation}

\section{Transfection efficiency and cellular toxicity}

To determine the optimum formulation strategy and amount of targeting peptide leading to the highest gene expression and lowest cellular toxicity, the transfection efficiency of the p18-4-modified lipoplexes was evaluated in human malignant melanoma (A375) cell line. Different concentrations of p18-4 modified gemini surfactant or free p18-4 (5, $10,15,20 \%$ and $100 \%$ of the total amount of cationic lipid) were tested. The highest levels of gene expression (IFN- $\gamma$ level by ELISA) were observed when $10 \%$ of p18-4 conjugated gemini surfactant (p18-4-G) or physical co-formulation of free p18-4 was used [Figure $3 \mathrm{~A}]$. ]. Lipoplexes formulated with
$100 \%$ of p18-4 conjugated gemini surfactant (p18-4$\mathrm{G})$ showed a negligible level of gene expression [data not shown]. Incorporation of p18-4 modified gemini surfactant in the lipoplexes (either during the formation of lipoplexes, L:2 Ch[P.G.p18-4-G.L] , or after the formation of [P.L.G] lipoplexes, L3: Ch[P.G.L].p18-4-G) resulted in a 3-fold increase in IFN- $\gamma$ level compared to standard non-targeting lipoplexes [P.G.L] [Figure 3 A]. The highest level of gene expression was obtained when lipoplexes were formulated by physical co-formulation of free p18-4 (L4: Ph[P.G.p18-4.L] and L5: Ph[P.G.L].p18-4) with over a 6-fold increase being observed $\left(1252 \pm 402\right.$ and $1152 \pm 328$ pg IFN- $\gamma / 1.5 \times 10^{4} \mathrm{~A} 375$ cells, respectively) [Figure $3 \mathrm{~A}$ ]. 

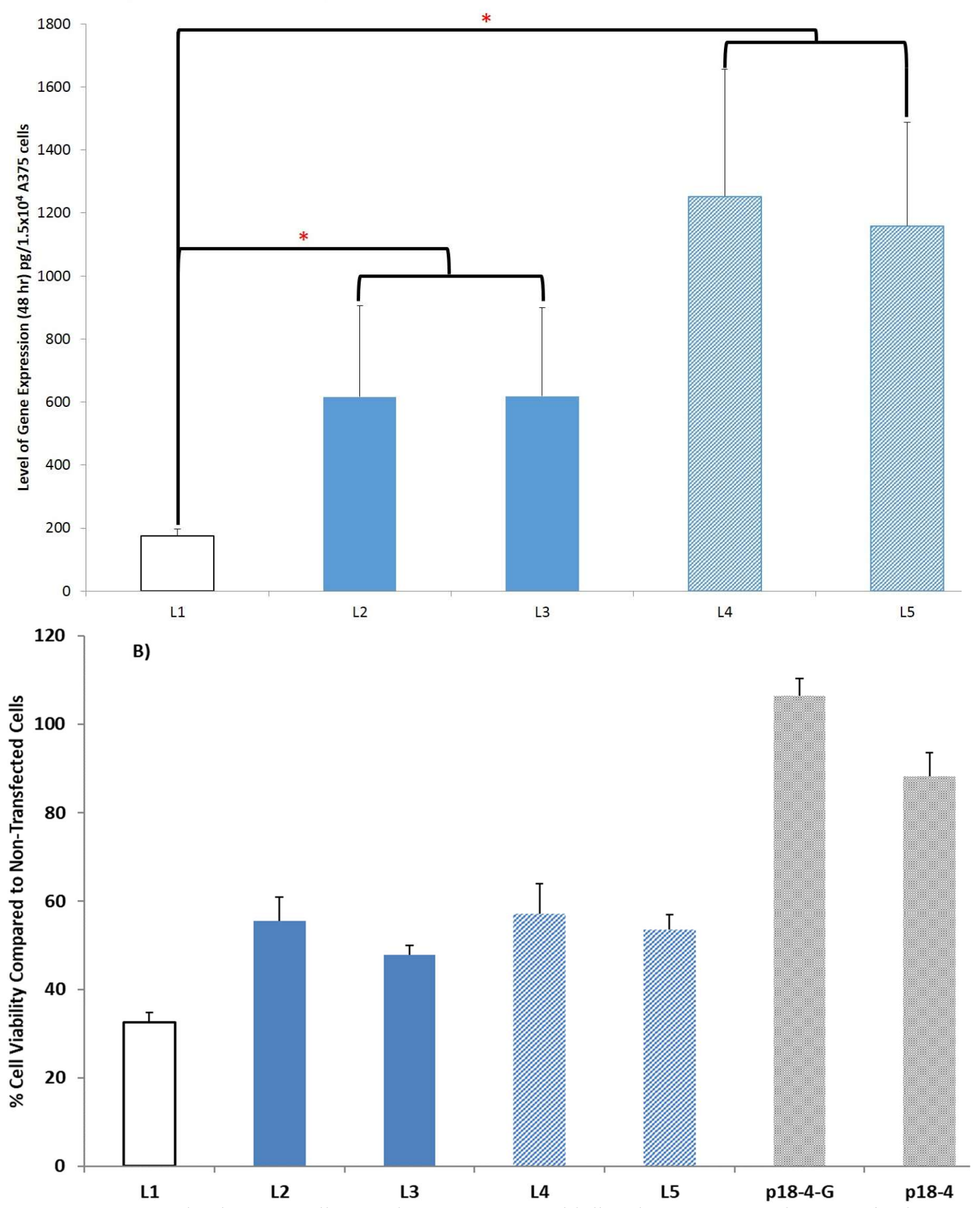

Figure 3 (A) IFN- $\gamma$ expression in A375 cells at 48-h post-treatment with lipoplexes constructed at 1:10 -/+ charge ratio. P: pDNA, G:12-7N(GK)-12, L: DOPE, p18-4-G : 12-7N( p18-4)-12, p18-4: p18-4 linear peptide. (Ch) indicates lipoplexes chemically conjugated p18-4-G and (Ph) lipoplexes physically co-formulated with free p18-4. IFN- $\gamma$ level determined by ELISA. Significant increases (* $\mathrm{p}<0.01$, one-way ANOVA) in IFN- expression were observed in all p18-4 modified lipoplexes compared to non-modified lipoplexes (L1:[P.G.L]). (B) Cell viability in the same A375 cells after a 48-h treatment with p18-4-modified lipoplex formulations as determined by MTT assay. Cell viability values are given as \% relative to nontransfected cells. Four wells of each formulations were loaded on three different plates. The results are expressed as the mean of the three plates $(n=3)$. Bars represent standard deviation. * Indicates significance at $\mathrm{p}<0.05$ in comparison to standard formulation [P.G.L] (L1) 
The influence of targeting peptide on the cellular toxicity of the lipoplexes was assessed by MTT assay [Figure $3 \mathrm{~B}$ ]. 48-h post-transfection, the cell viability for non-modified lipoplexes L1:[P.G.L] was approximately $\mathbf{3 0 \%}$ of the non-transfected cells [Figure $3 \mathrm{~B}$ ]. Higher cell viability values were reported for lipoplexes formed with $10 \%$ chemically modified gemini surfactant (L2: Ch[P.G.p18-4G.L] and L3:Ch[P.G.L].p18-4-G). Physical coadministration of free p18-4 with also showed an improvement in cellular viability compared to standard lipoplexes. Both p18-4 modified gemini surfactant (p18-4-G) and free targeting peptide (p184) caused no or minimal toxicity to A375 cell line [Figure $3 \mathrm{~B}$ ].

\section{Targeting efficiency}

The specificity of p18-4 modified lipoplexes to target melanoma cell lines was examined by transfecting primary human epidermal keratinocytes (HEKa) with standard lipoplexes, p18-4-G modified lipoplexes and lipoplexes co-formulated with free p18-4 (L1: [P/G/L], L2: Ch[P.G.p18-4-G.L] and L5: Ph[P.G.L].p18-4) [Figure 4 A]. The IFN- $\gamma$ level after 48 h of transfection with L2: Ch[P.G.p18-4G.L] was similar to the level achieved by standard non-modified lipoplexes L1: [P/G/L]. Transfection with L5: Ph[P.G.L].p18-4 showed a slight, but not significant $(p>0.05)$, increase in expressed IFN- $\gamma$ level compared to standard [P.G.L] lipoplexes.

MTT assay showed that targeting lipoplexes marginally enhanced the cellular viability in HEKa cell lines in comparison to standard [P.G.L] lipoplexes [Figure $4 \mathrm{~B}$ ].

\section{DISCUSSION}

Gene therapy for cancer is the most extensively explored amongst worldwide gene therapy clinical trials as $64.6 \%$ ( 1,590 trials) are investigating the potential of cancer gene therapy.(23) Creating an efficient and targeted gene delivery agent with effective therapeutic outcomes and good safety profile are required for successful clinical applications. Targeting tumors with lipid-based gene delivery systems can be achieved by chemically modifying the lipoplexes with tumor specific ligands.(24-26) Gemini surfactant-based lipoplexes have been widely explored for gene delivery as they show good transfection efficiency and low cellular toxicity. The glycyl-lysine modified gemini surfactant used in this work, $12-7 \mathrm{~N}(\mathrm{GK})-12$, showed potential application as a non-invasive topical transfection agent for development of vaginal genetic vaccination.(15) In addition, by chemically modifying the $12-7 \mathrm{~N}(\mathrm{GK})-12$ with cRGD, to target over-expressed integrin receptor on melanoma, led to a significant increase of gene expression.(17) The ability of the RGD-modified lipoplexes to specifically target overexpressed $\alpha 3 / \beta 1$ integrin in melanoma was confirmed by using a positive competitor peptide (free RGD) and false-negative control peptide (RAD). As well, the RGD-modified gemini surfactant lipoplex did not enhance the transfection activity in normal human epidermal keratinocytes (HEKa), indicating the selectivity of the system toward the target.(17).

The nature of the nano-carriers, such as modification of the chemical structure, and formulation strategies can influence the physicochemical properties of the lipoplex system, ultimately affecting the transfection efficiency. The size, shape and overall charge of the assembled lipoplexes can influence the physicochemical stability of the gene delivery system, its biodistribution, cellular uptake, gene expression, and cellular toxicity.(27) Gemini surfactant-based lipoplexes [P.L.G] can be internalized by mammalian cells via clathrin-mediated or caveolaemediated endocytosis pathways.(28) Modification of the chemical structure of the gemini surfactant influenced the shape of the lipoplexes and hence the main endocytosis uptake mechanism. For example, lipoplexes build with $12-7 \mathrm{NH}-12$ gemini surfactant formed spherical-shape nanoparticles and were internalized mainly through a clathrin-mediated pathway. On the other hand, the modification of the gemini surfactant with a dipeptide moiety in the spacer region (i.e., $12-7 \mathrm{~N}(\mathrm{GK})-12$ ) resulted in the formation of cylindrically shaped lipoplexes with larger particle size. This lipoplex system demonstrated $\mathrm{pH}$ sensitive characteristics and internalized the cells through caveolae-mediated endocytosis.(28) In the current work, co-formulation of lipoplex systems with free p18-4 (L4: Ph[P.G.p18-4.L] and L5: Ph[P.G.L].p18-4) showed the highest transfection efficiency with a 6fold increase in IFN- $\gamma$ level compared to standard lipoplexes L1: [P.G.L] [Figure 3 A]. The lipoplexes assembled in larger nanoparticles $(>150 \mathrm{~nm})$ with a polymodal particle distribution compared to the standard lipoplexes (L1 [P.G.L]). 

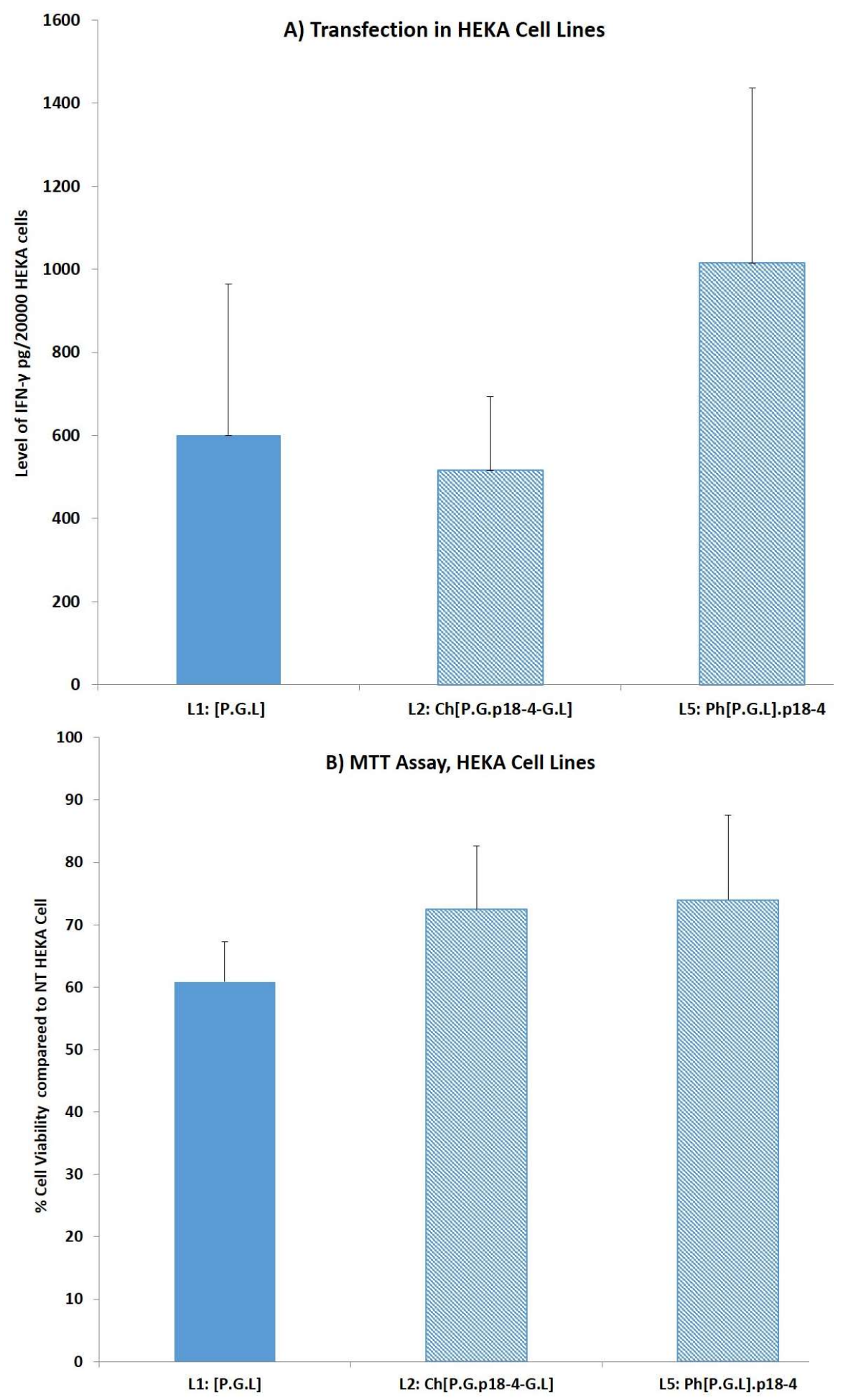

Figure 4 (A) IFN- $\gamma$ expression in HEKA cell line at 48-h post-treatment with lipoplexes constructed at 1:10 -/+ charge ratio non-modified lipoplexes (L1:[P.G.L]), p18-4 chemically-conjugated lipoplexes (L2: Ch[P.G.p18-4-G.L]) and p18-4 peptide co-formulated with the lipoplexes (L5: Ph[P.G.L].p18-4). IFN- $\gamma$ level determined by ELISA. (B) Cell viability of HEKA cells after a 48-h treatment with p18-4-modified lipoplex formulations as determined by MTT assay. Cell viability values are given as \% relative to non-transfected cells. HEKA cell line is normal adult human epidermal keratinocyte cells which express normal level of integrins. No statistical significates was observed between non-modified lipoplexes and p18-4- modified lipoplexes. Four wells of each formulations were loaded on three different plates. The results are expressed as the mean of the three plates $(n=3)$. Bars represent standard deviation. 
The heterogeneity of the size of these lipoplexes might have contributed to alterations in cellular uptake mechanisms, leading to an improvement in gene expression.(29) The modifications of the lipoplexes with targeting p18-4 peptide did not influence the supramolecular assembly, as all lipoplexes evaluated in this work arranged into inverted hexagonal structures $\left(\mathrm{H}_{\mathrm{II}}\right)$ [Figure 2]. This lipid phase arrangement is believed to contribute to the ability of the gemini based lipoplexes to interact with the cell membrane and facilitate the endosomal escape of the pDNA upon endocytosis.(13, 30)

The use of peptide penetration enhancers has been widely explored to enhance the uptake of highly hydrophobic drugs and in gene delivery. For skin delivery, these peptides interact with skin proteins (namely keratins which are highly expressed in the skin) and enhance the transcellular uptake of drug molecules.(31) In normal skin conditions, keratins (a family of intermediate filaments) are the main structural proteins in the epidermis (mainly type II keratin KRT1 and KRT10 which mainly expressed in keratinocytes) and they show important role in maintaining skin integrity and in intracellular signaling pathways.(32) Mutations in keratins expressions are contributed to several skin hereditary disorders.(32, 33) In addition, several keratins showed distinctive expression (upregulated or downregulated) in certain cancers (such as lung, breast and skin cancers) and can be used for disease diagnosis and monitoring.(32) The linear dodecapeptide peptide p18-4 is able to bind preferably to cancerous cells (MDA-MB-435 and MCF-7) and internalized upon binding through endocytosis. $(9,10)$ In a MDA-MB-435 xenograft mouse model, p18-4 conjugated doxorubicin showed a 5-fold reduction in tumor size compared to the free drug. $(9,10)$ p18-4 specifically interacts with overexpressed KRT-1 in MDA-MB-435 and MCF-7 cells.(34) In the current work, we evaluated the ability of p18-4 modified lipoplexes to target melanoma using a model cell line (A375) showing overexpression of keratins. $(35,36)$ The increase in gene expression by the p18-4 modified lipoplexes could be attributed to the specific interaction of $\mathrm{p} 18$ 4 with keratins and lead to improvement of cellular uptake of the lipoplexes and higher transfection activity [Figure 3 A]. We believe that the combination of heterogeneity of particle size and the availability of free p18-4 (not covalently bound to gemini surfactant) on the surface of the lipoplexes to interact with keratins in formulations L4 and L5 can explain the significant increase in transfection compared to non-modified (L1) and chemically modified lipoplexes (L2 and L3). The results of the control study (the transfection in HEKa cells which express a normal level of keratins) indicate the targeting specificity of the p18-4 modified lipoplexes to melanoma [Figure $4 \mathrm{~A}$ ].

\section{CONCLUSIONS}

The results of this study show that gemini surfactant based lipoplexes modified with targeting peptide (p18-4) are able to deliver pDNA to melanoma and achieve higher gene expression with a good safety profile. The specificity of melanoma targeting for the lipoplexes was demonstrated by the higher gene expression compared to non-malignant keratinocytes. The ability of the targeted lipoplexes to interact specifically to overexpressed keratins in melanoma and to enhance their penetration into the tissue indicate the potential application of the system for topical gene delivery.

\section{ACKNOWLEDGEMENTS}

We thank the help of Dr. Jackson Chitanda with the synthesis of gemini surfactants. Funding was provided by the College of Pharmacy and Nutrition, Natural Sciences and Engineering Council of Canada. Scholarship W M-S was provided by Taibah University, Saudi Arabia.

\section{CONFLICT OF INTEREST}

The authors declare that they have no conflict of interest.

\section{REFERENCES}

1. Greish K. Enhanced permeability and retention (EPR) effect for anticancer nanomedicine drug targeting. Cancer Nanotechnology: Springer; 2010. p. 25-37.

2. Danhier F, Feron O, Préat V. To exploit the tumor microenvironment: passive and active tumor targeting of nanocarriers for anti-cancer drug delivery. Journal of Controlled Release. 2010;148(2):135-46.

3. Bertrand N, Wu J, Xu X, Kamaly N, Farokhzad OC. Cancer nanotechnology: the impact of passive and active targeting in the era of modern cancer biology. Advanced Drug Delivery Reviews. 2014;66:2-25.

4. Steichen SD, Caldorera-Moore M, Peppas NA. A review of current nanoparticle and targeting moieties 
for the delivery of cancer therapeutics. European Journal of Pharmaceutical Sciences. 2013;48(3):41627.

5. Cheng Z, Al Zaki A, Hui JZ, Muzykantov VR, Tsourkas A. Multifunctional nanoparticles: cost versus benefit of adding targeting and imaging capabilities. Science. 2012;338(6109):903-10.

6. Zhang J, Spring H, Schwab M. Neuroblastoma tumor cell-binding peptides identified through random peptide phage display. Cancer Letters. 2001;171(2):153-64.

7. Askoxylakis V, Zitzmann S, Mier W, Graham K, Krämer S, von Wegner F, et al. Preclinical evaluation of the breast cancer cell-binding peptide, p160. Clinical Cancer Research. 2005;11(18):6705-12.

8. Soudy R, Gill A, Sprules T, Lavasanifar A, Kaur K. Proteolytically stable cancer targeting peptides with high affinity for breast cancer cells. Journal of Medicinal Chemistry. 2011;54(21):7523-34.

9. Shahin M, Soudy R, El-Sikhry H, Seubert JM, Kaur K, Lavasanifar A. Engineered peptides for the development of actively tumor targeted liposomal carriers of doxorubicin. Cancer Letters. 2013;334(2):284-92.

10. Shahin M, Soudy R, Aliabadi HM, Kneteman N, Kaur K, Lavasanifar A. Engineered breast tumor targeting peptide ligand modified liposomal doxorubicin and the effect of peptide density on anticancer activity. Biomaterials. 2013;34(16):408997.

11. Bombelli C, Giansanti L, Luciani P, Mancini G. Gemini surfactant based carriers in gene and drug delivery. Current Medicinal Chemistry. 2009;16(2):171-83.

12. Wettig SD, Verrall RE, Foldvari M. Gemini surfactants: a new family of building blocks for nonviral gene delivery systems. Current Gene Therapy. 2008;8(1):9-23.

13. Badea I, Verrall R, Baca-Estrada M, Tikoo S, Rosenberg A, Kumar P, et al. In vivo cutaneous interferon- $\gamma$ gene delivery using novel dicationic (gemini) surfactant-plasmid complexes. The Journal of Gene Medicine. 2005;7(9):1200-14.

14. Badea I. Gemini cationic surfactant-based delivery systems for non-invasive cutaneous gene therapy: University of Saskatchewan; 2006.

15. Singh J, Michel D, Getson HM, Chitanda JM, Verrall RE, Badea I. Development of amino acid substituted gemini surfactant-based mucoadhesive gene delivery systems for potential use as noninvasive vaginal genetic vaccination. Nanomedicine. 2015;10(3):40517.

16. Alqawlaq S, Sivak JM, Huzil JT, Ivanova MV, Flanagan JG, Beazely MA, et al. Preclinical development and ocular biodistribution of geminiDNA nanoparticles after intravitreal and topical administration: towards non-invasive glaucoma gene therapy. Nanomedicine: Nanotechnology, Biology and Medicine. 2014;10(8):1637-47.

17. Mohammed-Saeid W, Chitanda J, Al-Dulaymi M, Verrall R, Badea I. Design and evaluation of RGDmodified gemini surfactant-based lipoplexes for targeted gene therapy in melanoma model. Pharmaceutical Research. 2017;34(9):1886-96.

18. Yang P, Singh J, Wettig S, Foldvari M, Verrall RE, Badea I. Enhanced gene expression in epithelial cells transfected with amino acid-substituted gemini nanoparticles. European Journal of Pharmaceutics and Biopharmaceutics. 2010;75(3):311-20.

19. Michel D, Chitanda JM, Balogh R, Yang P, Singh J, Das U, et al. Design and evaluation of cyclodextrinbased delivery systems to incorporate poorly soluble curcumin analogs for the treatment of melanoma. European Journal of Pharmaceutics and Biopharmaceutics. 2012;81(3):548-56.

20. Al-Dulaymi MA, Chitanda JM, Mohammed-Saeid W, Araghi HY, Verrall RE, Grochulski P, et al. DiPeptide-Modified Gemini Surfactants as Gene Delivery Vectors: Exploring the Role of the Alkyl Tail in Their Physicochemical Behavior and Biological Activity. The AAPS journal. 2016:1-14.

21. Gratton SE, Ropp PA, Pohlhaus PD, Luft JC, Madden VJ, Napier ME, et al. The effect of particle design on cellular internalization pathways. Proceedings of the National Academy of Sciences. 2008;105(33):116138.

22. Rejman J, Oberle V, Zuhorn IS, Hoekstra D. Sizedependent internalization of particles via the pathways of clathrin-and caveolae-mediated endocytosis. Biochemical Journal. 2004;377(Pt 1):159.

23. Gene Therapy Clinical Trials Worldwide: John Wiley and Sons Ltd.; 2016 [cited 2012 25, April]. The Journal of Gene Medicine Clinical Trial site]. Available from: http://www.wiley.com//legacy/wileychi/genmed/clin ical/.

24. Harvie P, Dutzar B, Galbraith T, Cudmore S, O'Mahony D, Anklesaria P, et al. Targeting of lipidprotamine-DNA (LPD) lipopolyplexes using RGD motifs. Journal of Liposome Research. 2003;13(34):231-47.

25. Bruckheimer E, Harvie P, Orthel J, Dutzar B, Furstoss K, Mebel E, et al. In vivo efficacy of folatetargeted lipid-protamine-DNA (LPD-PEG-Folate) complexes in an immunocompetent syngeneic model for breast adenocarcinoma. Cancer Gene Therapy. 2003;11(2):128-34.

26. Surace C, Arpicco S, Dufaÿ-Wojcicki Al, Marsaud Vr, Bouclier Cl, Clay D, et al. Lipoplexes targeting the CD44 hyaluronic acid receptor for efficient transfection of breast cancer cells. Molecular Pharmaceutics. 2009;6(4):1062-73. 
27. Prabha S, Arya G, Chandra R, Ahmed B, Nimesh S. Effect of size on biological properties of nanoparticles employed in gene delivery. Artificial Cells, Nanomedicine, and Biotechnology. 2016;44(1):83-91.

28. Singh J, Michel D, Chitanda JM, Verrall RE, Badea I. Evaluation of cellular uptake and intracellular trafficking as determining factors of gene expression for amino acid-substituted gemini surfactant-based DNA nanoparticles. Journal of Nanobiotechnology. 2012;10(1):7.

29. Prabha S, Zhou W-Z, Panyam J, Labhasetwar V. Size-dependency of nanoparticle-mediated gene transfection: studies with fractionated nanoparticles. International Journal of Pharmaceutics. 2002;244(1):105-15.

30. Foldvari M, Badea I, Wettig S, Verrall R, Bagonluri M. Structural characterization of novel gemini nonviral DNA delivery systems for cutaneous gene therapy. Journal of Experimental Nanoscience. 2006;1(2):165-76.

31. Kumar S, Zakrewsky M, Chen M, Menegatti S, Muraski JA, Mitragotri S. Peptides as skin penetration enhancers: mechanisms of action. Journal of Controlled Release. 2015;199:168-78.
32. Moll R, Divo M, Langbein L. The human keratins: biology and pathology. Histochemistry and Cell Biology. 2008;129(6):705.

33. Roth W, Kumar V, Beer H-D, Richter M, Wohlenberg C, Reuter U, et al. Keratin 1 maintains skin integrity and participates in an inflammatory network in skin through interleukin-18. Journal of Cell Science. 2012;125(22):5269-79.

34. Soudy R, Etayash H, Bahadorani K, Lavasanifar A, Kaur K. Breast Cancer Targeting Peptide Binds Keratin 1: A New Molecular Marker for Targeted Drug Delivery to Breast Cancer. Molecular Pharmaceutics. 2017;14(3):593-604.

35. Clauser KR, Hall SC, Smith DM, Webb JW, Andrews LE, Tran HM, et al. Rapid mass spectrometric peptide sequencing and mass matching for characterization of human melanoma proteins isolated by twodimensional PAGE. Proceedings of the National Academy of Sciences. 1995;92(11):5072-6.

36. Hendrix MJ, Seftor EA, Chu Y-W, Seftor RE, Nagle RB, McDaniel KM, et al. Coexpression of vimentin and keratins by human melanoma tumor cells: correlation with invasive and metastatic potential. JNCI: Journal of the National Cancer Institute. 1992;84(3):165-74. 